\title{
Safety of transrectal ultrasound-guided prostate biopsy in patients affected by Crohn's disease
}

\author{
Lucio Dell'Atti, Andrea Benedetto Galosi \\ Department of Urology, University Hospital "Ospedali Riuniti”, Ancona, Italy.
}

\begin{abstract}
Summary Purpose: Crohn's disease (CD) is a chronic inflammatory condition of the gastrointestinal tract. It is usually considered a contraindication to transrectal ultrasound-guided prostate biopsy (TRUSBX). The aim of this study was to investigate the safety of TRUSBx in a small cohort of patients with CD.

Methods: We queried our institutional database clinical data of patients with a diagnosis of CD undergoing TRUSBX, and a retrospective prospective study of 5 patients was planned. All patients enrolled were in the remission phase of $C D$ and asymptomatic. They received the same antibiotic prophylaxis and a povidone-iodine aqueous solution enema before the procedure. A standardized reproducible technique was used with using a ultrasound machine equipped with a 5-9 $\mathrm{MHz}$ multifrequency convex probe "end-fire". The patients were treated under local anaesthesia, and a 14-core biopsy scheme was performed in each patient as first intention. After the procedure each patient was given a verbal numeric pain scale to evaluate tolerability of TRUSBx.

Results: TRUSBx was successfully completed in all patients. The number of biopsy cores was 14 (12-16). Of the 5 biopsy procedures performed $40 \%$ revealed prostatic carcinoma (PCa) with a Gleason score $6(3+3)$. No patients required catheterization or admission to the hospital for adverse events after the procedure. The most frequent adverse event was hematospermia (60\%), while hematuria was present in $20 \%$ of patients and a minimal rectal bleeding in $20 \%$ of the patients. No patients reported severe or unbearable pain (score $\geq 8$ ). Conclusions: This study suggests that CD may not be an absolute contraindication to TRUSBx for prostate cancer detection, but still requires a careful patients selection.
\end{abstract}

KEY WORDS: Crohn's disease; Inflammatory bowel disease; Prostate biopsy; Ultrasonography; Prostate cancer; Complications.

Submitted 8 March 2017; Accepted 23 April 2017

\section{INTRODUCTION}

Crohn's disease (CD) is a chronic inflammatory condition of the gastrointestinal tract characterized by a relapsing-remitting clinical behaviour, which may involve the whole digestive tract from the oral cavity to the anus (1). Incidence of CD is highest during last decades in high income countries as United States and Europe with rates ranging between 10-20/100.000 (2). The aetiology of this disease remains unknown; however, it is supposed that a number of causes are implicated, including genetic factors, immune-regulatory defects, microbial exposure and sex hormones such as androgens $(3,4)$. Recent studies reported that androgens may regulate aspects of human immunity and also be associated with a statistically significant increased risk of inflammatory bowel disease (IBD) $(5,6)$. Prostate cancer (PCa) is an androgen-dependent pathologic condition whose anatomical location requires careful consideration in patients with CD (7). However, the detection of PCa is reliant on prostate biopsy for histological confirmation (8). Therefore, approximately $20-30 \%$ of men with CD will require a prostate biopsy within their lifetime, and compared with men non affected, they are at increased risk of developing complications following this procedure $(7,8)$. Although cancer detection rates or the complication rates between transrectal (TR) biopsy and transperineal (TP) biopsy are greatly debated $(9,10)$, in this retrospective study we analysed the use and the safety of transrectal ultrasound-guided prostate biopsy (TRUSBx) in a small cohort of five patients with CD.

\section{Materials AND Methods}

Between August 2007 and January 2017, we performed a retrospective review of 5 patients affected by CD who underwent TRUSBx for abnormal digital rectal examination (DRE), high prostate-specific antigen (PSA) levels $(\geq 4 \mathrm{ng} / \mathrm{ml}$ ), or both. Patients with a history of biopsy, surgical treatment for prostatic disease and incomplete clinical data were excluded from our study. All patients enrolled in the study were in the remission phase of CD and asymptomatic. All patients signed a consent form for TRUSBx. None of the patients was receiving anticoagulants or antiplatelet agents before the procedure.

All patients received the same antibiotic prophylaxis: 5 days of ciprofloxacin (500 mg twice daily) starting $24 \mathrm{~h}$ before the biopsy (11). Moreover, all patients received a povidone-iodine aqueous solution enema starting fifteen minutes before the TRUSBx (12). All procedures were performed in order to empty the bladder, since we believe that even the state of bladder repletion may be an element of discomfort during the performance of mapping biopsy. TRUSBx was performed with the patient in the left lateral decubitus using a General Electric Logiq 7 machine equipped with a $5-9 \mathrm{MHz}$ multi-frequency convex probe "end-fire". The patients were treated under 
local anaesthesia with Lidocaine Spray $10 \mathrm{gr} / 100 \mathrm{ml}$ applied two minutes before the procedure (13). After having images of the prostate and measurement of the volume, sampling was carried out with a 18-Gauge Tru-Cut needle powered by an automatic spring-loaded biopsy disposable gun. A 14-core biopsy scheme was performed in each patient, as first intention, including 2 basal samples (lateral and medial), 2 parasagittal samples (lateral and medial), 2 apical samples (lateral and medial), and 1 transitional zone sample on each side. After the procedure each patient was given a verbal numeric pain scale (VNS), which was designed with 0 representing absence of pain and 10 the maximum pain they perceived in life. Patients were discharged after a short period of observation (typically 60 minutes) and once urinated.

Patients were seen for follow-up 2 weeks after the biopsy and a full clinical outcomes of all cases were available. Descriptive statistics for variables with a normal distribution, non-normal distribution, and categorical variables were evaluated using mean and interquartile range, according to their distribution. Statistical analyses were performed using Microsoft Excel 2010 platform. A p < 0.05 was considered to indicate statistical significance.

\section{RESULTS}

Over the study period on 3,470 men underwent TRUSBx, 5 patients affected by CD in clinical and endoscopic remission were retrospectively enrolled in the study. The mean age of enrolled patients was 63 years (range 56-71), with a prostate volume of $51 \mathrm{ml}$ (range 39-60), initial PSA levels of $7.3 \mathrm{ng} / \mathrm{ml}$ (range 4.9-10.2). The clinical characteristics of the patients are shown in Table 1. TRUSBx was successfully completed in all patients. The number of biopsy cores was 14 (range 12-16). Of the 5 biopsy procedures performed $2(40 \%)$ revealed PCa with a Gleason score $6(3+3)$.

In the remain 3 patients 2 biopsies showed prostatitis, while 1 reported benign tissue. Patients with higher serum PSA appeared more likely to have cancer on TRUSBx $(p<0.001)$. No patients required catheterization or admission to the hospital for adverse events after the procedure. The most frequent adverse event was hematospermia (60\%), which had also the longest duration (24.6 days). Hematuria was present in $20 \%$ of patients and a minimal rectal bleeding was observed in 20\% of the patients after the biopsy (Figure 1). There were no serious infectious compli-

Table 1.

Figure 1. undergoing transrectal ultrasound prostate biopsy.

\begin{tabular}{|c|c|c|c|c|c|}
\hline Patients & 1 & 2 & 3 & 4 & 5 \\
\hline Age (yrs) & 56 & 62 & 59 & 67 & 71 \\
\hline Age at diagnosis (yrs) & 29 & 35 & 32 & 44 & 52 \\
\hline $\mathrm{BMI}\left(\mathrm{Kg} / \mathrm{m}^{2}\right)$ & 27.2 & 31 & 28.4 & 32.5 & 33 \\
\hline Race & Caucasian & Caucasian & Caucasian & Caucasian & Caucasian \\
\hline Smoking & Current & Past & Yes & Never & Past \\
\hline Positive family history PCa & No & No & Yes & No & No \\
\hline Localisation of CD & Ileum-colon & Colon & Ileum-colon & Colon & Ileum \\
\hline Prior surgery for $C D$ & No & No & No & No & Yes \\
\hline Prostate volume (ml) & 46 & 58 & 39 & 60 & 52 \\
\hline PSA level (ng/ml) & 5.6 & 4.9 & 10.2 & 6.9 & 8.5 \\
\hline $\mathrm{N}^{\circ}$ biopsy cores & 14 & 14 & 12 & 16 & 14 \\
\hline Abnormal DRE, (n) & No & Yes & Yes & No & No \\
\hline Prostate cancer, (n) & No & No & Yes & No & Yes \\
\hline \multicolumn{6}{|l|}{ Biopsy Gleason score, (n) } \\
\hline$\leq 6$ & 0 & 0 & 1 & 0 & 1 \\
\hline 7 & 0 & 0 & 0 & 0 & 0 \\
\hline$\geq 8$ & 0 & 0 & 0 & 0 & 0 \\
\hline Pain in VNS & 3 & 2 & 7 & 2 & 3 \\
\hline
\end{tabular}

cations in this study. The mean pain in the visual numerical scale in patients was $3.4(2-7)$. No patients reported severe or unbearable pain (score $\geq 8$ ).

\section{Discussion}

Crohn's disease is a IBD characterised by recurrent and destructive inflammation of the gastrointestinal tract $(2,3)$. Approximately $35 \%$ of IBD are diagnosed with an extra-intestinal manifestation of the disease, including uveitis, erythema nodosum, peripheral arthritis and sclerosing cholangitis (14).

Inflammation is known to play an important role in carcinogenesis, and there is also a growing evidence that patients with $\mathrm{CD}$ have an increased risk for intestinal

The prevalence of adverse events in patients with Crohn's disease undergoing transrectal ultrasound prostate biopsy.

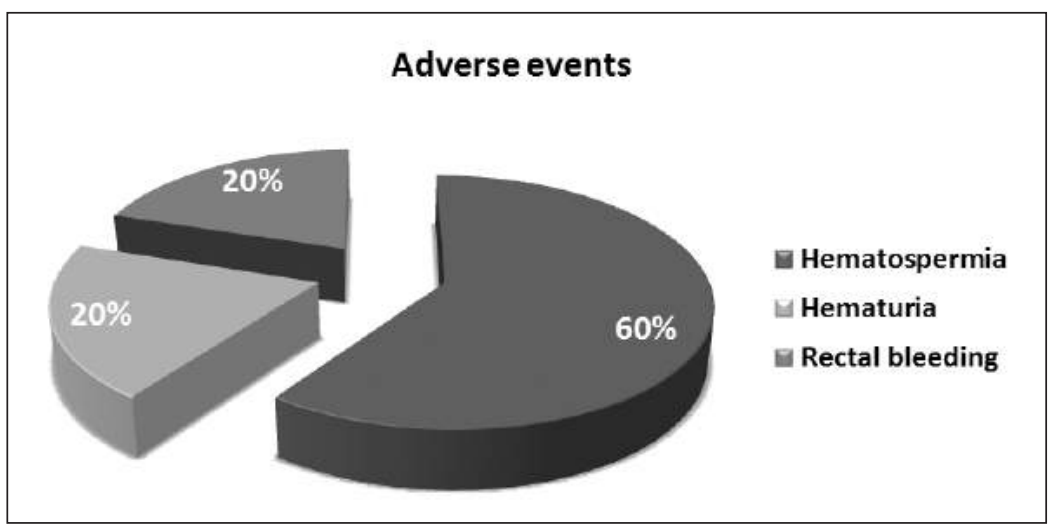


cancers secondary to long-standing intestinal inflammation or secondary to immunosuppressive therapies (15, 16). While it is well established that androgens play a role in PCa development, there are new evidences that androgens may also regulate aspects of human immunity (6).

Moreover, Klil-Dori et al. reported that the suppression of androgens to castration levels in a large population cohort of patients affected by PCa was associated with a decreased risk of IBD (7). In line with this study, the androgen deprivation through surgical castration has been shown to protect the intestinal permeability in a porcine model (17) and influence the composition of murine gut microbiota (18). Prostate biopsy plays an important role in diagnosing $\mathrm{PCa}$, confirming the grade of cancer and stratifying cancer aggressiveness (8). In 20$30 \%$ of patients with $\mathrm{CD}$, a prostate biopsy will be require within their lifetime, and compared with patients with other benign intestinal conditions, they are at increased risk of developing postoperative complications following prostate biopsy (19). Some studies reported several risk factors in patients with CD following intestinal surgery for postoperative morbidities, including the presence of perforating disease, prolonged duration of symptoms, weight loss, anaemia, malnutrition and several acute flares of CD (19-21). Patients with rectal disease such as $C D$ represent a population in which the standard technique of TRUSBx may not be feasible for postoperative morbidities listed above. However, in literature, the comparisons between the TR and TP prostate biopsy approaches showed no significant difference in the overall cancer detection rate and in the incidence of major or minor complications $(10,22)$. To our knowledge, this is the first study that report the largest series to date of TRUSBX in patients affected by CD. Fergany and Angermeier described the technique of TP prostate biopsy using the ultrasound grid and a stabilizing platform employed for prostate brachytherapy in patient with ulcerative colitis and an ileal pouch. In their case study multiple cores of prostatic tissue were successfully obtained without major complications (23). Compared with TR biopsy, TP approach may offer a more aseptic method, with fewer infective complications in light of evidence of fluoroquinolone-resistant bacteria in bowel. However, TP biopsy is a more costly alternative, with longer procedural times and higher risks of acute retention postoperatively $(9,10,22,24)$. In our Department we usually employ a TR approach using an end-fire probe, because we believe that to perform prostate biopsy in the anterior area of the gland it is the most appropriate technique.

The end-fire probe permits biopsy cores to be taken more transversely (oriented along an anterior-posterior axis), has a more oblique-angled trajectory, and more flexibility in manoeuvring the biopsy direction than sidefire probes (25). In this population of patients TRUSBx provided a good visualization of the gland and facilitated multiple random needle biopsies under local anaesthesia without major complications. The most frequent adverse event was hematospermia (60\%), hematuria and a rectal bleeding were observed in $20 \%$ of patients after the procedure, respectively. However, in this study several limi- tations need to be acknowledged such as the use of a retrospective analysis, enrolling a small patient population, and omitting the preoperative evaluation of clinical symptoms. Therefore, the present study is not sufficiently powered to assess the impact of improved prostate biopsy technique on outcomes in patients with CD.

\section{Conclusions}

In conclusion, our study suggests that that CD may not be an absolute contraindication to TRUSBx for PCa detection, but still requires a careful patients selection. This case series is a unique, highly selected group of patients affected by $C D$ in clinical remission, further studies to elucidate the best biopsy practices and share decision-making are required.

\section{References}

1. Leighton JA, Pasha SF. Inflammatory Disorders of the Small Bowel. Gastrointest Endosc Clin N Am. 2017; 27:63-77.

2. Molodecky NA, Soon IS, Rabi DM, et al. Increasing incidence and prevalence of the inflammatory bowel diseases with time, based on systematic review. Gastroenterology. 2012; 142:46-54.

3. Xavier RJ, Podolsky DK. Unravelling the pathogenesis of inflammatory bowel disease. Nature. 2007; 448:427-34.

4. Loftus EV Jr. Clinical epidemiology of inflammatory bowel disease: Incidence, prevalence, and environmental influences. Gastroenterology. 2004; 126:1504-17.

5. Fijak $M$, Meinhardt A. The testis in immune privilege. Immunol Rev. 2006; 213:66-81.

6. Morse MD, McNeel DG. Prostate cancer patients on androgen deprivation therapy develop persistent changes in adaptive immune responses. Hum Immunol. 2010; 71:496-504.

7. Klil-Drori AJ, Tascilar K, Yin H, et al. Androgen Deprivation Therapy and the Incidence of Inflammatory Bowel Disease in Patients With Prostate Cancer. Am J Epidemiol. 2016; 184:15-22.

8. Epstein JI, Sanderson H, Carter HB et al. Utility of saturation biopsy to predict insignificant cancer at radical prostatectomy. Urology. 2005;66:356-60.

9. Scott S, Samaratunga H, Chabert $C$, et al. Is transperineal prostate biopsy more accurate than transrectal biopsy in determining final Gleason score and clinical risk category? A comparative analysis. BJU Int. 2015; 116:26-30.

10. Shen PF, Zhu YC, Wei WR, et al. The results of transperineal versus transrectal prostate biopsy: a systematic review and metaanalysis. Asian J Androl. 2012; 14:310-5.

11. Wu XW, Ji HZ, Wang FY. Meta-analysis of ciprofloxacin in treatment of Crohn's disease. Biomed Rep. 2015; 3:70-4.

12. Hay JM, Boussougant Y, Lacaine F, et al. Povidone-iodine enema as a preoperative bowel preparation for colorectal surgery. A bacteriologic study. Dis Colon Rectum. 1989; 32:9-13.

13. Dell'Atti L. Lidocaine spray administration in transrectal ultrasound-guided prostate biopsy: five years of experience. Arch Ital Urol Androl. 2014; 86:340-3.

14. Feuerstein JD, Flier SN, Yee EU, et al. A rare case series of concomitant inflammatory bowel disease, sporadic adenomas, and serrated polyposis syndrome. J Crohns Colitis. 2014; 8:1735-9. 
15. Axelrad JE, Lichtiger S, Yajnik V. Inflammatory bowel disease and cancer: The role of inflammation, immunosuppression, and cancer treatment. World J Gastroenterol. 2016; 22:4794-801.

16. Ullman TA, Itzkowitz SH. Intestinal inflammation and cancer. Gastroenterology. 2011; 140:1807-16.

17. Deitch EA, Senthil M, Brown M, et al. rauma-shock-induced gut injury and the production of biologically active intestinal lymph is abrogated by castration in a large animal porcine model. Shock. 2008; 30:135-41.

18. Markle JG, Frank DN, Mortin-Toth S, et al. Sex differences in the gut microbiome drive hormone-dependent regulation of autoimmunity. Science. 2013; 339:1084-8.

19. Bernell O, Lapidus A, Hellers G. Risk factors for surgery and recurrence in 907 patients with primary ileocaecal Crohn's disease. Br J Surg. 2000; 87:1697-701.

20. Iesalnieks I, Kilger A, Glass H, et al. Intraabdominal septic complications following bowel resection for Crohn's disease: detrimental influence on long-term outcome. Int J Colorectal Dis. 2008; 23:1167-74.

21. Dindo D, Demartines N, Clavien PA. Classification of surgical complications: a new proposal with evaluation in a cohort of 6336 patients and results of a survey. Ann Surg. 2004; 240:205-13.

22. Shida Y, Hakariya T, Takehara K, et al. Comparison Between a Combined Transrectal and Transperineal Approach and a Transrectal Approach for Prostate Rebiopsy. Anticancer Res. 2016; 36:4685-90.

23. Fergany AF, Angermeier KW. A technique of transrectal ultrasound guided transperineal random prostate biopsy in patients with ulcerative colitis and an ileal pouch.J Urol. 2000; 163:205-6.

24. Abdollah F, Novara G, Briganti A, et al. Trans-rectal versus trans-perineal saturation rebiopsy of the prostate: is there a difference in cancer detection rate? Urology. 2011; 77:921-5.

25. Galosi AB, Tiroli M, Cantoro D, et al. Biopsy of the anterior prostate gland: technique with end-fire transrectal ultrasound. Arch Ital Urol Androl. 2010; 82:248-52.

\section{Correspondence}

Lucio Dell'Atti, MD, PhD (Correspondant Author)

dellatti@hotmail.com

Andrea Benedetto Galosi, MD

Department of Urology - Marche Polytechnic University

University Hospital "Ospedali Riuniti"

71 Conca Street - 60126 Torrette, Ancona, Italy 